\title{
Carpal valgosity in foals
}

\section{BERNARD TUREK, KATARZYNA SIEWRUK, OLGA DREWNOWSKA, ZDZISŁAW GAJEWSKI}

\author{
Division of Large Animal Surgery, *Division of Animal Reproduction, Andrology and Biotechnology of Reproduction, \\ Department of Large Animal Diseases with Clinic, Faculty of Veterinary Medicine, \\ Warsaw University of Life Sciences -SGGW, Nowoursynowska 100, 00-797 Warsaw, Poland
}

\section{Turek B., Siewruk K., Drewnowska O., Gajewski Z. Carpal valgosity in foals \\ Summary}

A three-week-old Arabian female foal and a three-week-old Silesian male foal were diagnosed with carpal valgosity confirmed by radiographic evaluation. In both cases, the wire bridge method was used for treatment. After the operation, mobility restriction was recommended. Even though the foals differed significantly in weight, the outcome was good. This confirms that the wire bridge method is an effective method of treating carpal valgosity and leads to fast recovery (within 3-4 weeks).

Keywords: foal, carpal joint, deformity, treatment, wire bridge

The carpal joint is the most prone to dysarthrosis in foals. The main causative factors include genetic predisposition, breed and prenatal environment (placenta, parasitological status, metabolic disorders and colic), as well as insufficient welfare, including the feeding type (4). The correct diagnosis of carpal valgosity (carpus valgus) is possible after a basic clinical examination, but the essential diagnosis can only be confirmed by an $\mathrm{x}$-ray, which helps recognize the exact location and advancement of the angular deformation, as well as exclude the congenital disorders of the carpal bone, such as its absence or malformation (1). It is also obligatory to determine whether rotational or contractual malformations coexist, which may result in subsequent treatment complications (6).

Bone growth and the resulting changes in limb alignment are a dynamic process, which continues until epiphyseal cartilage closure. Existing deformations should be corrected during this time. The most common corrective methods adjust the horizontal strength and bone growth direction until clinical improvement is achieved. The key to choosing an appropriate treatment method and to successful treatment is to know the detailed physiology of the epiphyseal cartilage, its inner growth activity and the closure time (5). After that time, more complicated methods, such as angular ostectomy and joint arthrodesis, may be applied, but only to save the animal's life. Further sports career is impossible (3).

The main types of carpal joint deformities are basically congenital and are easily diagnosed just after birth. Carpal joint deformities acquired later, during the bone growth stage and animal maturation, are caused essentially by irregular bone growth. The average physiological carpal angular bend is between 2 and 5 degrees. Most foals are born with a carpal bend that disappears during the bone growth stage (2) as a result of the progressive growth of the chest and its further gradual broadening, which causes thoracic limb inversion (1). The neonatal environment and welfare, feeding type and activity pattern are very important factors contributing to the acquired carpal changes. The main surgical methods decreasing the bone growth rate are a single transphyseal screw and a wire bridge. The first method is based on the application of a transphyseal cortical screw, $4.5 \mathrm{~mm}$ in diameter, at the 45-degree angle to the epiphyseal cartilage. After drilling and threading a screw canal, a transphyseal screw is introduced through the epiphyseal cartilage into the epiphysis of the radial bone at the convex from the metaphysis approach or, in the case of carpal valgosity, from the medial approach (1). In the second method, two cortical screws with a diameter of $4.5 \mathrm{~mm}$ are introduced parallel to the epiphyseal cartilage into the methaphysis and epiphysis to avoid accidental joint space penetration and cartilage damage. Next, wire loops are placed between the two screws, forming a wire bridge. The bone implants, irrespective of the treatment method selected, decrease bone growth at the convex and must be removed after limb angulation has improved. 


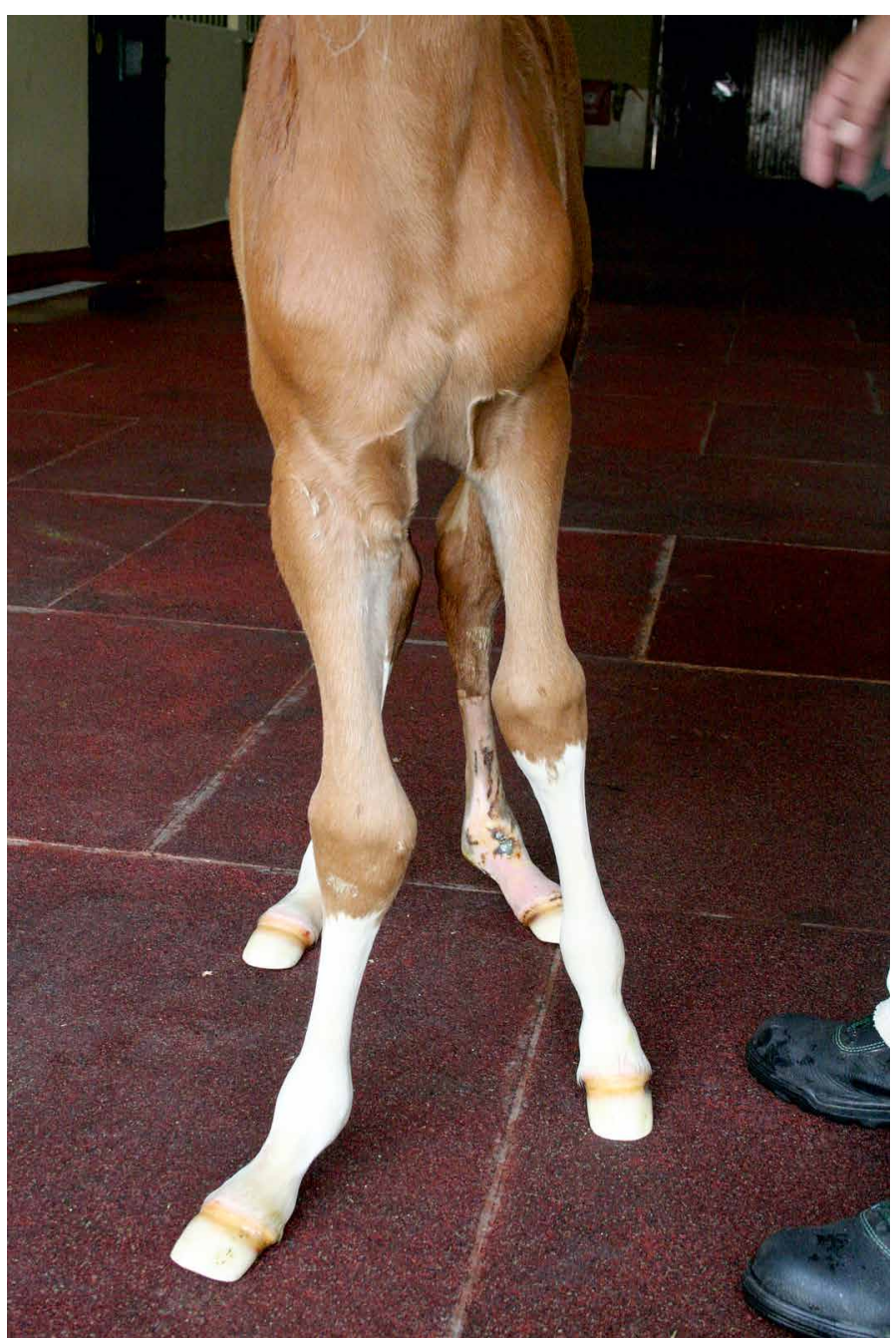

Fig. 1. Case 1 with carpus valgus (front right limb)

\section{Cases description}

Two newborn foals were orthopedically treated with the wire bridge method.

Case 1. A three-week-old Arabian horse female foal, $70 \mathrm{~kg}$ body weight, was born healthy and without any clinical disorders. The foal's growth and weight gain were appropriate. The only clinical sign detected was a slight medial, angular limb deformation at the carpal joint level (carpal valgosity). The deformation process also occurred in the autopodial region because of natural growth (Fig. 1). An x-ray examination was performed, and an epiphyseal cartilage enlargement of the distal radial bone and a medial angulation of the limb (at approximately 19 degrees) were found. A hard splint and mobility restriction were applied, but without any clinical improvement, so a surgery was scheduled.

An orthopedic surgery was performed under general anesthesia with isofurane following premedication, in the right lateral position. A semicircular skin incision from the medial approach was made. The target points for the cortical screws, $4.5 \mathrm{~mm}$ in diameter, were chosen after an $\mathrm{x}$-ray examination. The first screw was introduced into the distal radial epiphysis, and the other into the metaphysis. Loops between them were performed with wire of $1 \mathrm{~mm}$ in diameter (Fig. 2). The postoperative antibiotic treatment

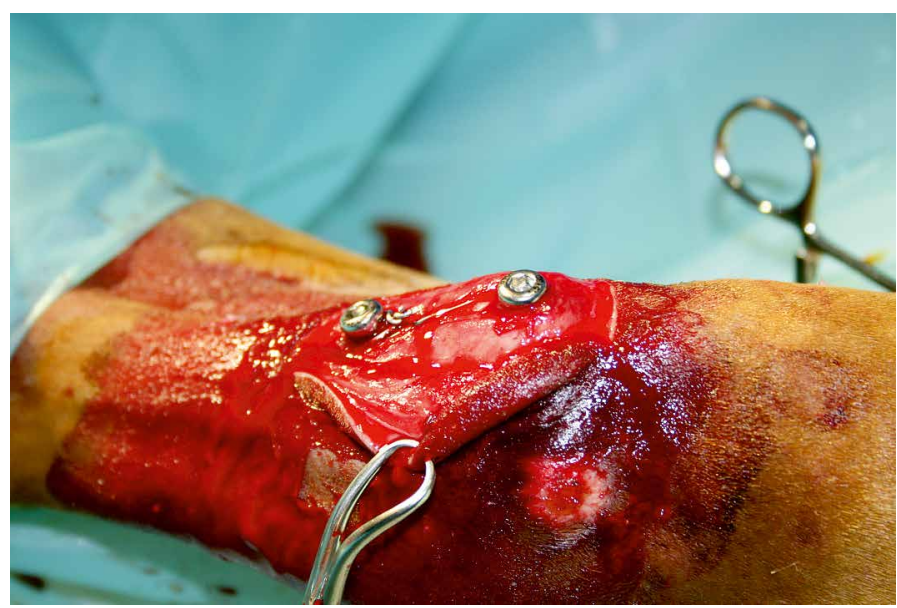

Fig. 2. Intraoperative view of the wire bridge applied in case 1

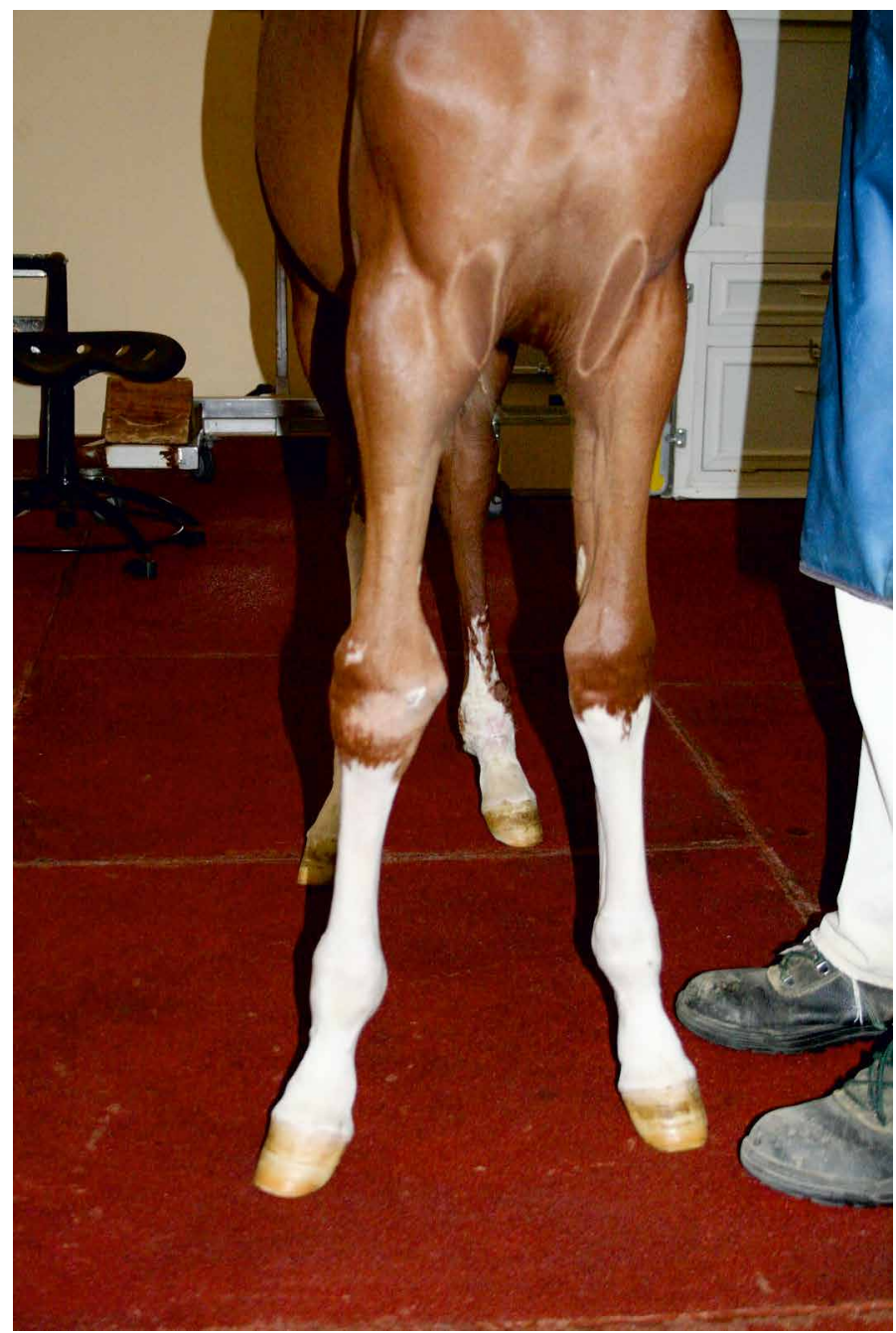

Fig. 3. View of the front legs (foal 1) after treatment

consisted of ceftiofur administered at a dose of $10 \mathrm{mg} / \mathrm{kg}$ every 12 hours and gentamicine at $6.6 \mathrm{mg} / \mathrm{kg}$ every 24 hours. The soft cast was changed after one week and removed after another. The limb angulation improved significantly after three weeks of the recommended mobility restriction (pen rest combined with walking) (Fig. 3). The bone implants were removed in the fourth week (Fig. 8).

Case 2. A three-week-old Silesian horse male foal weighing $150 \mathrm{~kg}$ was presented for orthopedic examination. The angulation of the left thoracic limb was visible from birth 


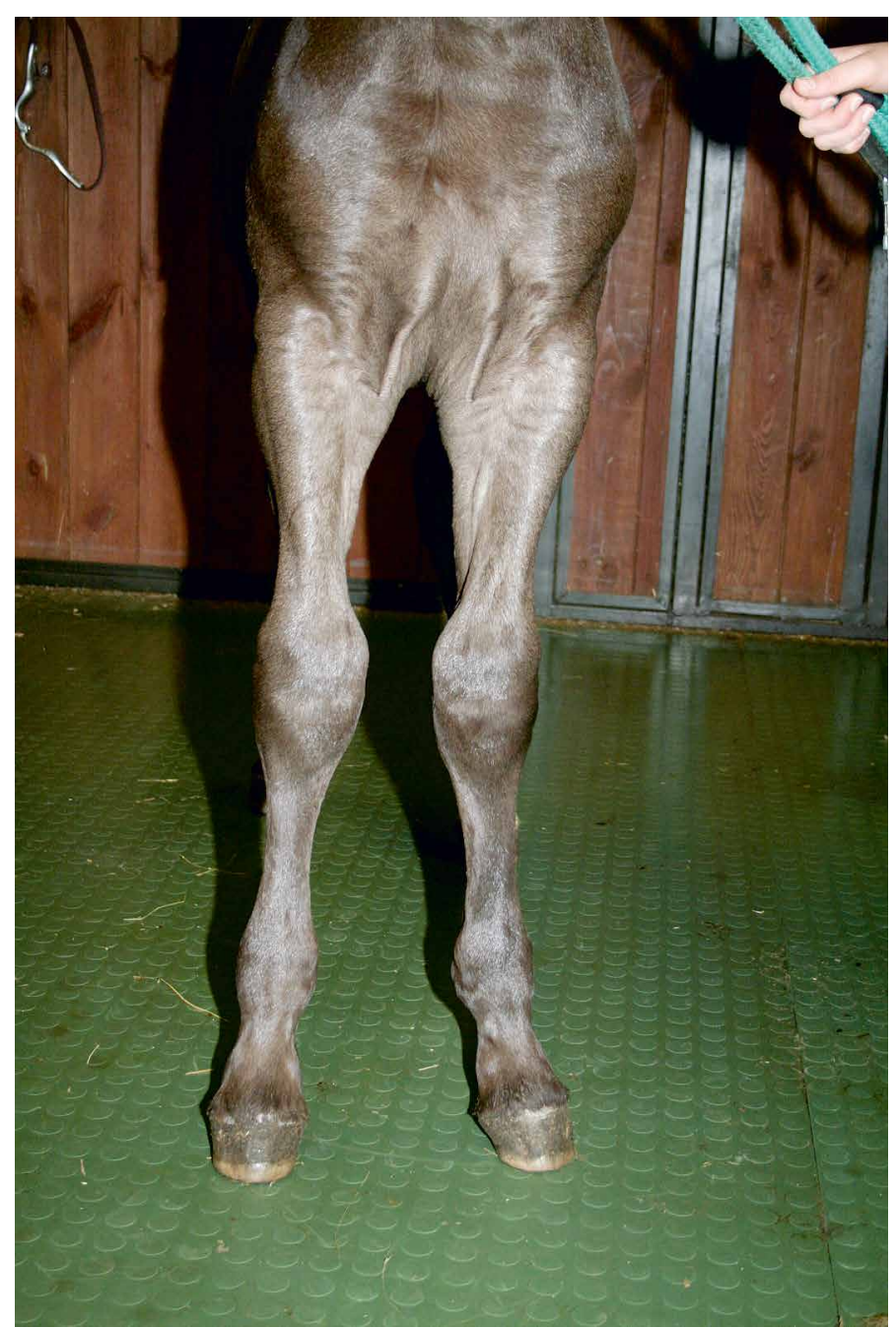

Fig. 4. Case 2 with carpus valgus (front left limb)

and increased with time (Fig. 4). An x-ray examination was performed (Fig. 5). It showed a significant epiphyseal cartilage enlargement of the distal radial bone with a medial angulation of the limb (of approximately 18 degrees) at the carpal joint level. A wire-bridge type surgery was scheduled. The wire bridge type surgery was performed up to the pattern. A postoperative antibiotic therapy was applied for the following five days with an intramuscular procaine penicillin injection at a dose of $24000 \mathrm{IU} / \mathrm{kg}$ combined with intravenously administered gentamicin at a dose of $6.6 \mathrm{mg} /$ $\mathrm{kg}$ once a day. The soft cast was changed after one week and removed after another. There were no complications during the wound healing process. The limb angulation improved significantly after three weeks (Fig. 6), as confirmed by an $\mathrm{x}$-ray examination in the cranio-caudal view (Fig. 7). The bone implants were removed after four weeks (Fig. 8).

\section{Discussion}

In both cases, carpal valgosity was confirmed after birth. The problem increased because of the animals' growth and weight gaining. No clinical improvement was observed after conservative treatment with movement limitation combined with a hard cast. The carpal valgosity diagnosed was less than 20 degrees. Where a greater angle is diagnosed, the chances of total clini-

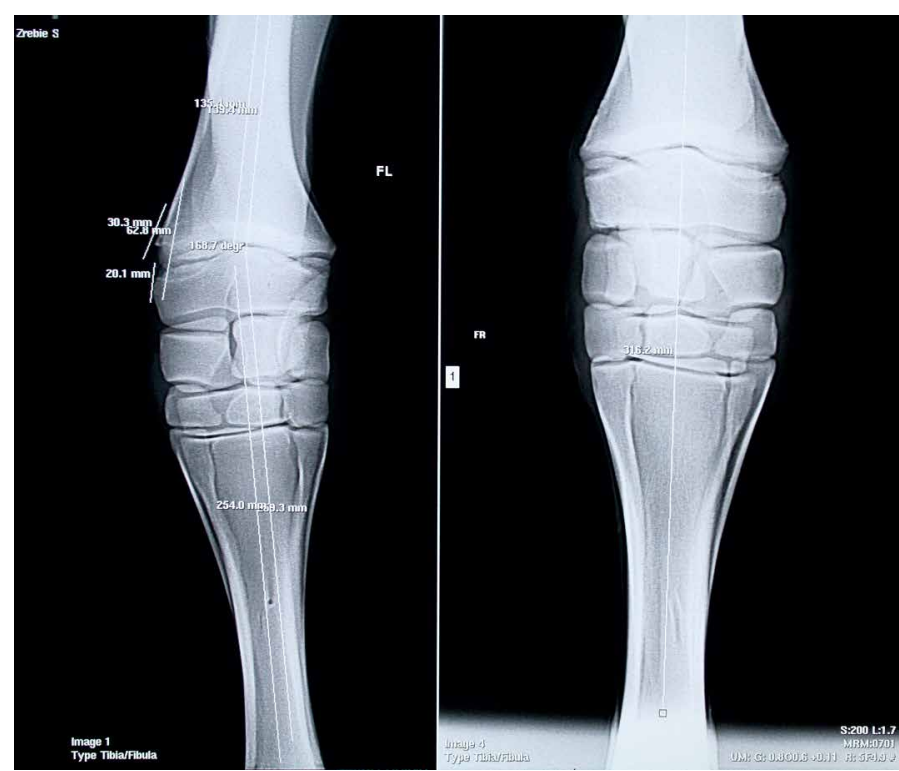

Fig. 5. X ray (ap view) of both carpal joints showing the deformation of the front left leg

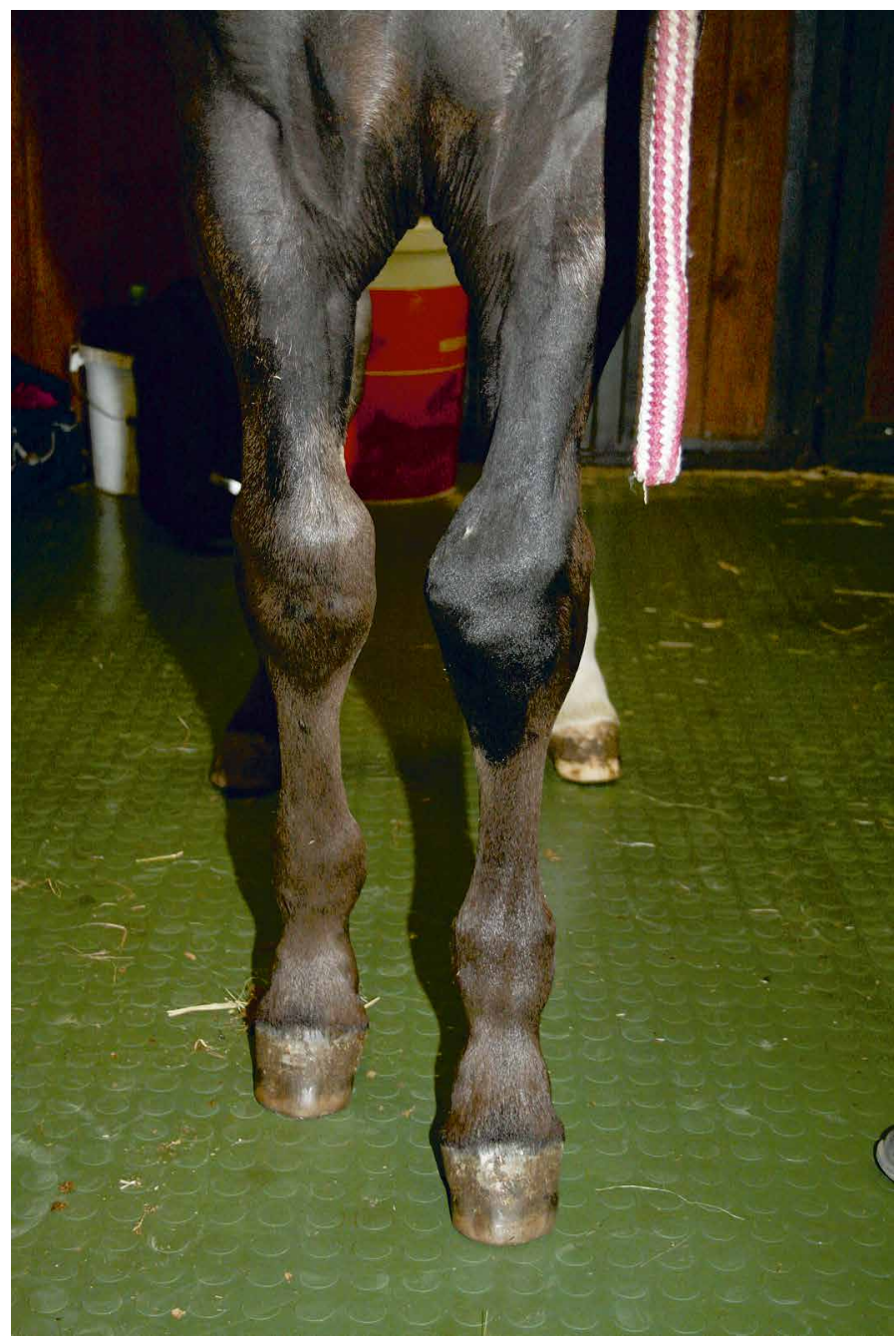

Fig. 6. View of the front legs (foal 2) after treatment

cal improvement are smaller, and the recuperation time is longer. It may be necessary to apply two surgical methods, such as the wire bridge at the convex with the periosteum separation at the opposite site. Since, the carpal valgosity diagnosed in both foals was less than 


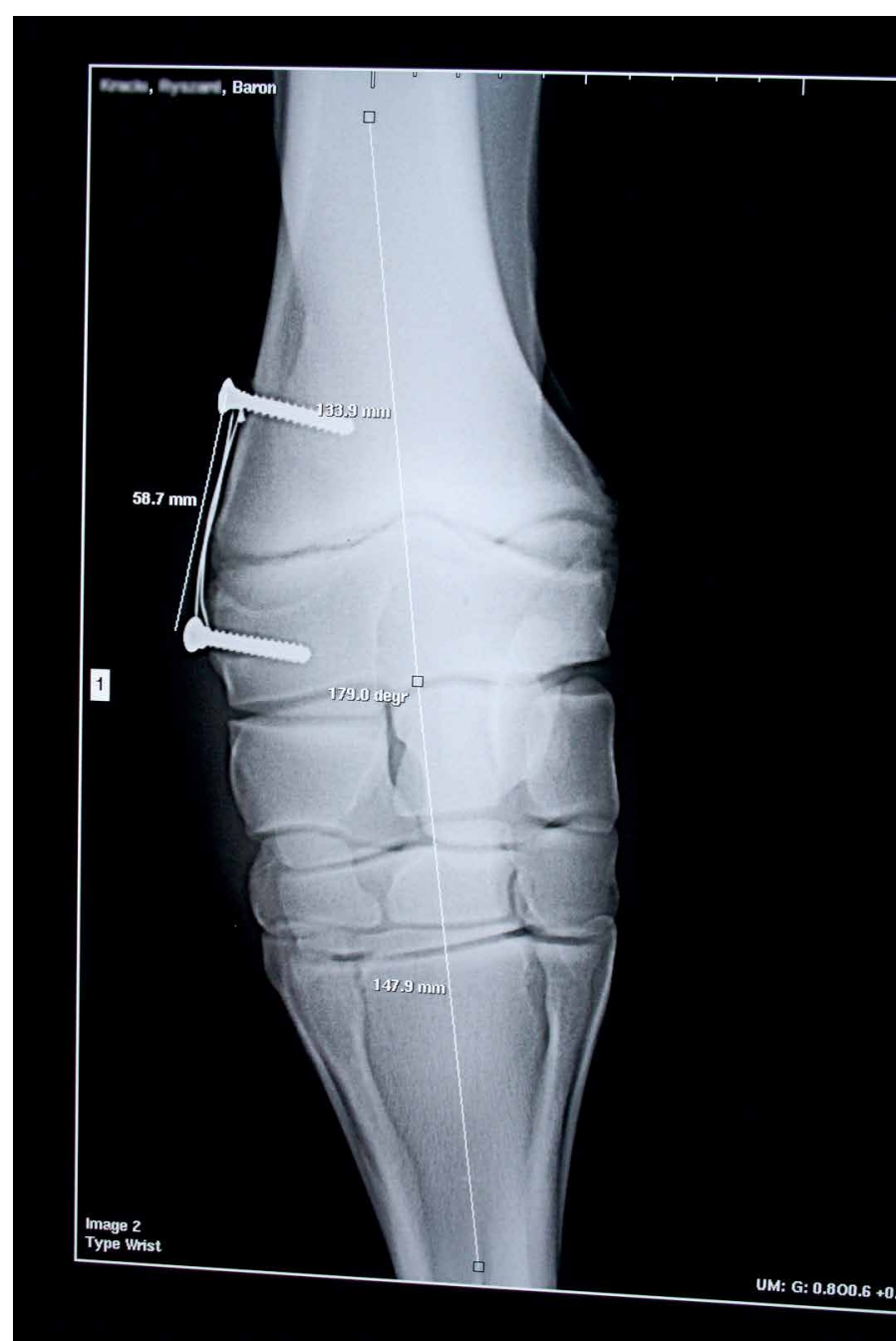

Fig. 7. Case 2. X ray (ap view) of the affected leg after treatment. The long axis of the leg is straight

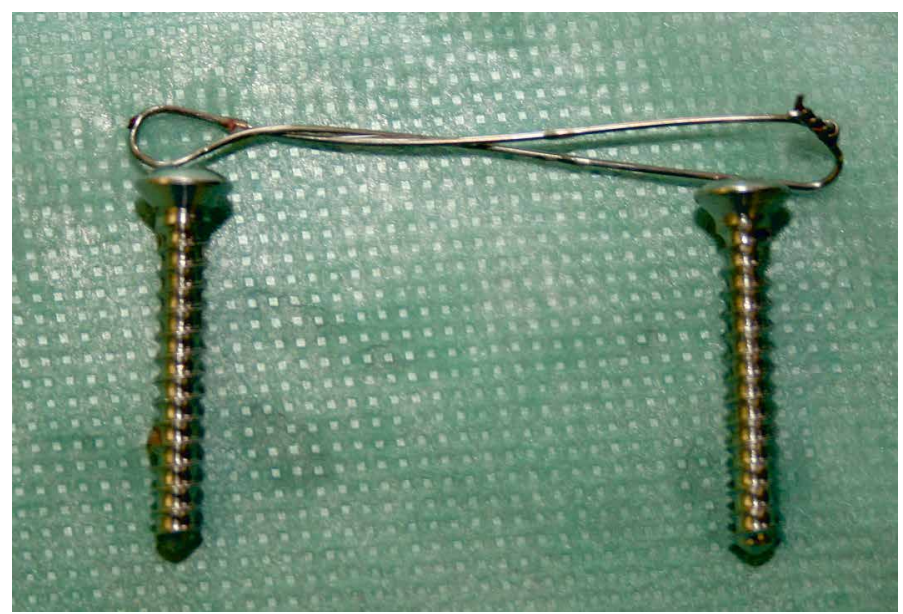

Fig. 8. Screws and wire removed after treatment

20 degrees, only the wire bridge method was applied to reduce bone growth at the convex. No complications were observed in postoperative wound healing, other than the enlargement of the capital epiphysis, cartilage and methaphysis resulting from chronic periosteitis caused by wire compression, which disappeared 3-4 months after the bone implants were removed.
Clinical experience was the key to treatment method selection. Methods considered included the introduction of a single cortical screw into the distal metaphysis through the epiphyseal cartilage up to the epiphysis and a periosteum incision at the opposite site to enhance bone growth. According to a 2012 research publication by Bramlage, the wire bridge method proved better as the main treatment method in similar cases. The single screw method was found to be less complicated and quicker, but resulted in remote clinical improvement to a smaller degree. The most common postoperative complication was epiphysitis in the single screw pattern, whereas postoperative wound infections were common after both patterns (1)

An excessive correction of the limb pattern was described as a common postoperative complication, which can be avoided with a follow up x-ray examination and the removal of bone implants at an appropriate time. The epiphysitis diagnosed was a transient problem.

Postoperative mobility restriction is a pivotal stage, allowing the limb to straighten. If the mobility restriction period is too short, pathological changes may ensue in the postoperative period, particularly in coldblooded breed foals such as Silesian horses because of their rapid weight gain, almost twice as fast as that of Arabian foals.

The wire bridge method is less complicated and more effective as a carpal valgosity treatment. With an angle of less than 20 degrees, the recovery time is about three weeks for limb extension. The postoperative complications, such as epiphysitis and metaphysitis, were found to be transient and subsided a few months after the bone implants had been removed.

\section{References}

1. Bramlage L. R., Auer J. A.: Diagnosis, assessment and treatment strategies for angular limb deformities in the foal. Clin. Tech. Equine Pract. 2006, 5, 259-269.

2. Carlson E. R., Bramlage L. R., Stewart A., Embertson R. M., Ruggles A. J., Hopper S. A.: Complications after two transphyseal bridging techniques for treatment of angular limb deformities of the distal radius in 568 thoroughbred yearlings. Equine Vet. J. 2012, 44, 416-419.

3. Getman L. M.: Surgical treatment of severe, complex limb deformities in horses. Equine Vet. Educ. 2011, 23, 386-390.

4. Heidbrink U. H., Kühn S.: Surgical Treatment of carpus valgus - a retrospective evaluation. ECVS $15^{\text {th }}$ Annual Scientific Meeting, June $29^{\text {th }}-$ July $1^{\text {st }}, 2006$, Sevilla, Spain.

5. Whitfield-Cargile C., Watkins J. P.: Bilateral distal radial epiphysectomy and pancarpal arthrodesis for correction of complex carpal deformities in an American miniature horse. Equine Vet. Educ. 2011, 23, 381-385.

6. Witte S., Hunt R.: A review of angular limb deformities. Equine Vet. Educ. 2009, 21, 378-387.

Corresponding author: Dr Katarzyna Siewruk, PhD, Nowoursynowska 100, 00-797 Warsaw, Poland; e-mail: siewruk.katarzyna@gmail.com 